\title{
Synthesis of hollow silica nanospheres with a microemulsion as the template $\dagger$
}

\author{
Yu-Shen Lin, ${ }^{a}$ Si-Han Wu, ${ }^{a}$ Chih-Ta Tseng, ${ }^{a}$ Yann Hung, ${ }^{a}$ Chen Chang ${ }^{b}$ \\ and Chung-Yuan Mou*a
}

Received (in Cambridge, UK) 9th February 2009, Accepted 2nd April 2009

First published as an Advance Article on the web 6th May 2009

DOI: $10.1039 /$ b902681a

\begin{abstract}
We demonstrate a sol-gel approach, using a water-in-oil microemulsion as the template, for the synthesis of hollow and yolk/shell silica nanospheres, which can encapsulate pre-formed hydrophobic nanoparticles, and we then explore these multifunctional hollow nanospheres in cell-labeling applications.
\end{abstract}

Hollow spherical silica nanoparticles are a class of nanomaterials with potential applications in catalysis, separation, cell-labeling and drug delivery. ${ }^{1,2}$ The encapsulation of agents of various functionalities, such as photoactive, magnetic or catalytic properties, inside the hollow spheres would increase their potential applications. Hollow particles have been prepared from many templates, such as polystyrene spheres, ${ }^{3}$ emulsion droplets ${ }^{4,5}$ or vesicular structures. ${ }^{6}$ However, most of them are above $200 \mathrm{~nm}$ in size as in emulsion or vesicle templates. The large size is usually not effective for biomedical applications since cell uptake is limited. Another important requirement in biomedical application of nanoparticles is their aqueous suspensibility. For silica materials, the easy silica condensation reaction during the sol-gel process or calcination poses problems in making the particles sticking to each other to form bigger particles. Often, the high temperature removal of the template poses problems in solution suspension or destruction of encapsulated agents. Furthermore, after the hollow spheres are made, encapsulations of bigger agents such as enzymes or nanoparticles are usually difficult unless very delicate ship-in-bottle syntheses can be designed.

Recently, nanosized AlOOH hollow spheres were synthesized using a water-in-oil (W/O) microemulsion system. ${ }^{7}$ $\mathrm{W} / \mathrm{O}$ microemulsion offers advantages in giving uniform sub-100 nm hollow spheres because the small $\mathrm{W} / \mathrm{O}$ droplets are thermodynamically stable and thus uniform. Microemulsions can, in principle, provide one with uniform nanosized oil $(\mathrm{O} / \mathrm{W}$ type) or water $(\mathrm{W} / \mathrm{O})$ droplets for templating. Little success in obtaining hollow silica spheres has been achieved before. One tends to obtain just solid spheres instead. ${ }^{8}$ The formation of hollow silica spheres by a soft templating scheme depends on two crucial factors: (1) the stability of the surfactant organization, and (2) interfacial deposition of silica to form the nanoshell. Silica condensation

${ }^{a}$ Department of Chemistry, National Taiwan University, Taipei, 106, Taiwan.E-mail: cymou@ntu.edu.tw; Fax: + 886 23660954;

Tel: + 886233665251

${ }^{b}$ Institute of Biomedical Sciences, Academia Sinica, Taipei, 115,

Taiwan

$\dagger$ Electronic supplementary information (ESI) available: Experimental details and additional figures. See DOI: 10.1039/b902681a is often too facile to be limited to the very small interfacial region. One needs a way to decrease the extent of silica condensation in the center of spheres.

We would like to report here a novel method, based on templating microemulsion, for the synthesis of nanosized hollow spheres of silica with encapsulated magnetic nanoparticles for biolabeling applications. Herein we chose to study a particular reverse microemulsion system, consisting of a surfactant, Triton X-100, a co-surfactant, hexanol, and aminopropyltrimethoxy silane (APTS) with tetraethoxysilane (TEOS) being the silica sources. This system gives a stable microemulsion at room temperature. Moreover, the microemulsion can contain pre-made oil-soluble nanoparticles. This allows one a strategy of encapsulation of them inside the final hollow silica sphere. This method should be generally applicable to many oil-suspended nano-entities. In this paper, we demonstrate this strategy by encapsulating $\mathrm{Fe}_{3} \mathrm{O}_{4}$ magnetic nanoparticles. The magnetism would allow one the capacity of magnetic manipulation or MRI cell-imaging to track its whereabouts.

The $\mathrm{W} / \mathrm{O}$ reverse microemulsion system we used was made from cyclohexane, Triton X-100 (polyoxyethylene tert-octylphenyl ether), hexanol and water. This is a well-studied reverse microemulsion system stable to a wide range of changes in solution conditions. ${ }^{9}$ The preparation of the microemulsion is a slight modification of the method reported by Tan et al. ${ }^{10}$ We have designed two different mixing processes with the same surfactant system (Scheme 1). In process A, TEOS was initially hydrolyzed in ammonia to form $\mathrm{Si}(\mathrm{OEt})_{x}(\mathrm{OH})_{y}$ oligomer at the interface between water and oil, then APTS was added. During the process, the solution is transparent indicating very small nanoparticles $(<100 \mathrm{~nm})$ were formed in $\mathrm{W} / \mathrm{O}$ microemulsion. Only by ethanol wash one gets the precipitate to collect. One then washes repeatedly with water to obtain the hollow silica nanospheres (HS). In process B, APTS was added before the addition of ammonia. In the presence of excess APTS which has one less condensable group, the as-synthesized silica spheres are "soft". Removal of the core part occurs during repeated washing in water and dispersing with ultrasonication to form hollow spheres. Detailed procedures and compositions are given in ESI. $\dagger$

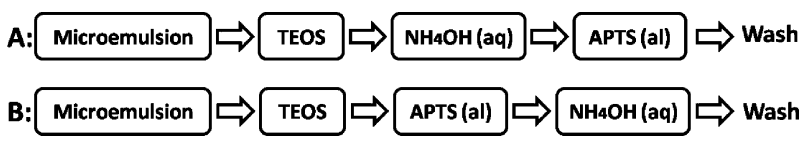

Scheme 1 


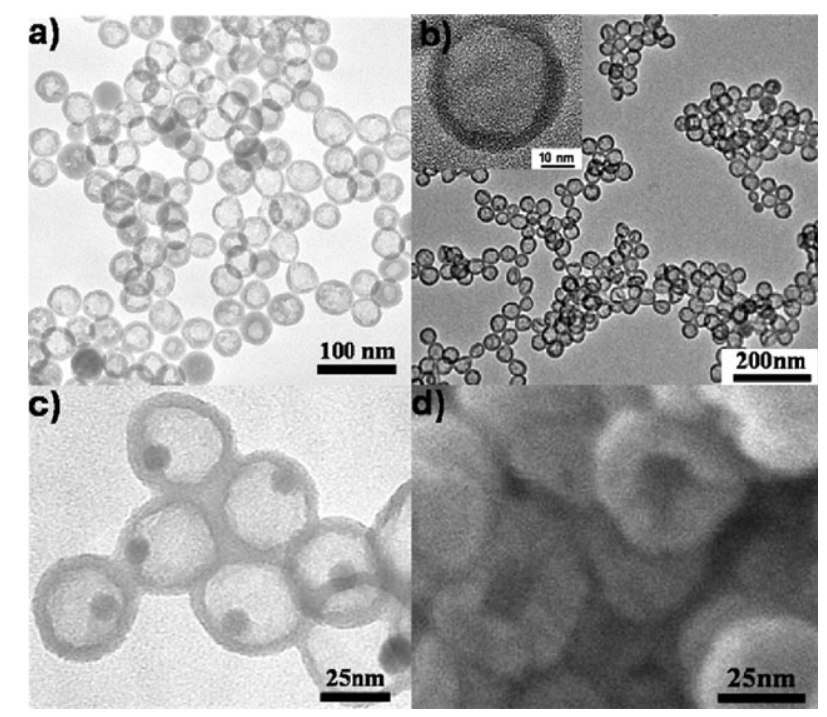

Fig. 1 TEM images of (a) hollow silica nanospheres, HS; (b) dyemodified hollow nanospheres, FITC-HS, prepared by process A; inset is a high-resolution TEM image showing the $5 \mathrm{~nm}$ shell thickness; (c) $\mathrm{Fe}_{3} \mathrm{O}_{4} @$ FITC-HS; size of $\mathrm{Fe}_{3} \mathrm{O}_{4}$ is $10 \mathrm{~nm}$; (d) SEM image of HS, a broken shell clearly shows the hollow interior.

For process A, typical results of TEM images showing the hollow silica nanospheres (HS), the dye-modified hollow silica nanosphere (FITC-HS), and $\mathrm{Fe}_{3} \mathrm{O}_{4}$ encapsulated FITC-HS $\left(\mathrm{Fe}_{3} \mathrm{O}_{4} @\right.$ FITC-HS) are shown in Fig. 1a-c. The inset in Fig. $1 \mathrm{~b}$ shows the spheres at about $40 \mathrm{~nm}$ in size and the internal diameter $30 \mathrm{~nm}$, while the wall thickness was about $5 \mathrm{~nm}$; the size distribution is quite uniform (data shown as Fig. S1 in ESI $\dagger$ ). Scanning electron microscopy (SEM) confirmed the hollow nature of the spheres (Fig. 1d). From the TEM image (Fig. 1c), it is obvious that $\mathrm{Fe}_{3} \mathrm{O}_{4}$ nanoparticles were encapsulated inside the hollow silica nanospheres. Small amounts of $\mathrm{Fe}_{3} \mathrm{O}_{4} @$ FITC-HS contain more than one $\mathrm{Fe}_{3} \mathrm{O}_{4}$ nanoparticle, but $\sim 90 \%$ of $\mathrm{Fe}_{3} \mathrm{O}_{4} @$ FITC-HS has just one iron oxide core.

$\mathrm{N}_{2}$ adsorption and desorption isotherms (Fig. S2a in ESI $\dagger$ ) show all were of type IV behavior with large hysteresis between $P / P_{\mathrm{o}}=0.45-0.9$. The BET surface area was $280 \mathrm{~m}^{2} \mathrm{~g}^{-1}$ for FITC-HS (compared to $72 \mathrm{~m}^{2} \mathrm{~g}^{-1}$ for solid spheres ${ }^{11}$ ) and $\mathrm{BJH}$ pore size distribution is around $30 \mathrm{~nm}$ (the inset of Fig. S2a in ESI $\dagger$ ) which agrees with the hollow interior volume as observed from TEM images. Also, we note that the step-like shape in the desorption curve near $P / P_{\mathrm{o}} \sim 0.75$ indicates some mesopores exist on the shell. This confirms the porous nature of the shell. There is little difference between HS and FITC-HS, but $\mathrm{Fe}_{3} \mathrm{O}_{4} @$ FITC-HS showed less surface area than HS, decreased from 280 to $224 \mathrm{~m}^{2} \mathrm{~g}^{-1}$. Wide angle $\mathrm{X}$-ray diffraction (XRD) confirmed that the crystalline nature of embedded $\mathrm{Fe}_{3} \mathrm{O}_{4}$ was retained after silica shell coating (Fig. S2b in ESI $\dagger$ ). The dye containing hollow silica nanoparticles, FITC-HS, appeared green under a hand-held illuminator. The photoluminescence spectrum of $\mathrm{Fe}_{3} \mathrm{O}_{4} @$ FITC-HS exhibited typical emission of FITC (Fig. S3a in ESI $\dagger$ ).

The iron content of $\mathrm{Fe}_{3} \mathrm{O}_{4} @$ FITC-HS nanoparticles was determined by inductively coupled plasma atomic emission spectroscopy (ICP-AES) to be $6.33 \mathrm{wt} \%$. This can

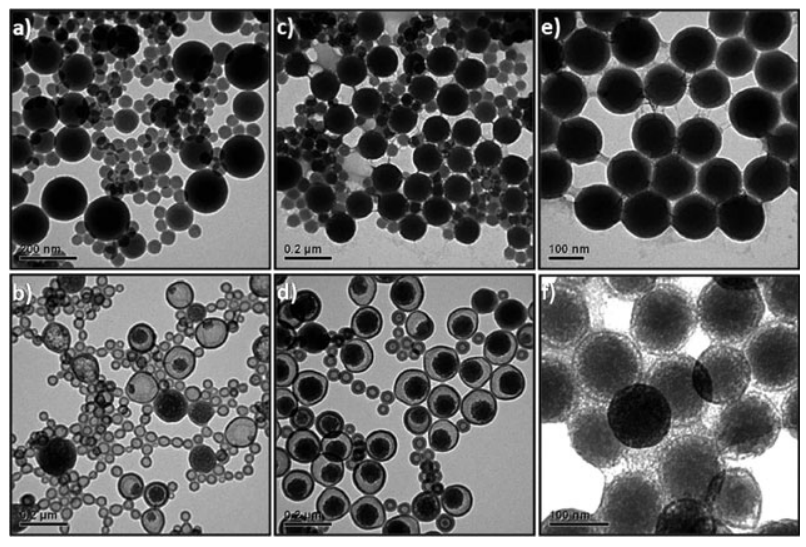

Fig. 2 TEM images of as-prepared silica nanospheres by process B with different amounts of APTS ethanolic solution: (a) $50 \mu \mathrm{L}$; (c) $100 \mu \mathrm{L}$; (e) $200 \mu \mathrm{L}$ and after soaking in $\mathrm{H}_{2} \mathrm{O}$ for a week (b) $50 \mu \mathrm{L}$; (d) $100 \mu \mathrm{L}$; (f) $200 \mu \mathrm{L}$.

be increased by using a higher amount of iron oxide. Magnetic behavior of the nanoparticle-incorporated hollow spheres was examined with SQUID (Fig. S3b in ESI $\dagger$ ). The saturation magnetization of $\mathrm{Fe}_{3} \mathrm{O}_{4} @$ FITC-HS remained high and displayed superparamagnetic behavior at room temperature, indicating these nanoparticles could serve as a $T_{2}$ MRI contrast agent. In addition, the discrete $\mathrm{Fe}_{3} \mathrm{O}_{4} @$ FITC-HS can be easily dispersed in water while the acid-treated magnetic hollow silica nanospheres from a $\mathrm{CaCO}_{3}$ template aggregated to a great extent. ${ }^{12}$ In process $\mathrm{A}$, the amount of TEOS/APTS ratio is critical; with too much TEOS, solid spheres formed. While, with too much APTS, the hollow spheres were destroyed during the washing step. The time lag for the addition of APTS is also important. Longer waiting leads to a thicker silica shell since more silica condensation occurs.

In process $\mathrm{B}$, after addition of APTS the solution became turbid, instead of transparent as in process $\mathrm{A}$, and a wide size distribution of silica spheres (from $30 \mathrm{~nm}$ to $160 \mathrm{~nm}$ ) was obtained. Fig. 2 presents the TEM images of three samples prepared with various amounts of APTS ethanolic solution. The upper row gives the images of as-synthesized sample at various amounts of APTS. The lower row gives the TEM images of the sample after they are soaked in water for about one week. It is observed that there are small hollow spheres $(40 \mathrm{~nm})$ and larger yolk/shell spheres $(\sim 100 \mathrm{~nm})$ (Fig. 2b and d). The shell thickness is estimated to be around $10 \mathrm{~nm}$ for both hollow and yolk/shell nanospheres. As the amount of APTS is increased, the proportion of yolk/shell structure and the size of yolk also increase. APTS is necessary for the formation of a HS structure. In both process $\mathrm{A}$ and $\mathrm{B}$, without the addition of APTS ethanolic solution, solid silica nanoparticles with uniform size of about $40 \mathrm{~nm}$ were obtained.

We would like to evaluate potential biomedical applications of $\mathrm{Fe}_{3} \mathrm{O}_{4} @$ FITC-HS obtained by process A. Like the solid core-shell SPIO@ $\mathrm{SiO}_{2}$ (FITC) nanoparticle previously reported by us, ${ }^{11}$ the hollow spheres can be internalized into many different cells such as HeLa, MCF-7 and NIH3T3, as evidenced by the confocal microscope images (Fig. 3a, for $\mathrm{HeLa}$ cells). Since the $\mathrm{Fe}_{3} \mathrm{O}_{4}$ nanoparticles inside the spheres retain their superparamagnetism and there is enough iron 


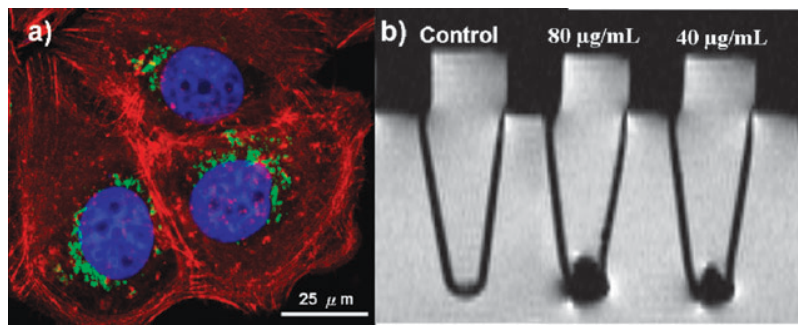

Fig. 3 (a) Confocal microscope image of HeLa cells uptake of $\mathrm{Fe}_{3} \mathrm{O}_{4} @$ FITC-HS; (b) $T_{2}$-weighted MR images of a pellet of $1 \times 10^{5}$ cells treated with 40 or $80 \mu \mathrm{g} \mathrm{mL}{ }^{-1} \mathrm{Fe}_{3} \mathrm{O}_{4} @$ FITC-HS for $1 \mathrm{~h}$, taken with a $4.7 \mathrm{~T}$ MR instrument.

content in the hollow spheres, the nanoparticle-labeled cells were subjected to MRI. Fig. $3 \mathrm{~b}$ is the phantom MRI images of $1 \times 10^{5}$ cells treated with different amounts of the nanoparticles, which showed a great contrast from control. Like the core/shell silica nanoparticles we previously reported, ${ }^{11}$ the MTT test did not show any acute adverse effect on the cell proliferation and viability (Fig. S4 in ESI $\dagger$ ).

Finally, we would like to make some comments on the possible mechanism of formation of hollow silica nanospheres and the incorporation of iron oxide nanoparticles. The use of $\mathrm{W} / \mathrm{O}$ type microemulsion is important because the silicas are formed inside the water droplet. This leaves the surface surfactant molecules still functioning to protect the silica nanopartciles from sticking to other nanospheres. In a separate experiment described in the ESI $\dagger$, we show there is roughly a linear correlation between the droplet sizes of microemulsion and the product particle size as measured by TEM, with the former always greater than the later (Table S1 in ESI $\dagger$ ). This result quantifies our hypothesis that the nanoparticles are formed inside the water domain in the $\mathrm{W} / \mathrm{O}$ microemulsion (details in ESI $\dagger$ ). A recent attempt to make hollow spheres using the $\mathrm{O} / \mathrm{W}$ microemulsion leads to sticking hollow spheres which are less useful for biomedicine. ${ }^{13}$ In their $\mathrm{O} / \mathrm{W}$ approach, TEOS condenses and grows outward from the oil-water interface and easily sticks to other colliding nanoparticles. In our approach, the interior void is created after prolonged washing. This is possible because (1) APTS has a less tendency to condense, and (2) the PEO group in Triton $\mathrm{X}-100$ binds strongly to the water in reverse microemulsion making silica condensation very limited. However, careful adjustment of the TEOS/APTS ratio and the duration of soaking and washing are important. During the synthesis, before the addition of TEOS to the mixture, the hydrophobic $\mathrm{Fe}_{3} \mathrm{O}_{4}$ nanoparticles reside in the oil phase. The hydrolyzed TEOS then replaces the surface protecting group of oleylamine to bring the $\mathrm{Fe}_{3} \mathrm{O}_{4}$ nanoparticles into the water phase, as shown recently by Koole et al. for the case of quantum dots in silica. ${ }^{14}$ The extent of $\mathrm{Fe}_{3} \mathrm{O}_{4}$ nanoparticle loadings can be adjusted easily. Using the same principle, other kinds of nanoparticles could be incorporated to the hollow nanospheres, $\mathrm{Au}$ and $\mathrm{TiO}_{2}$ for example. ${ }^{15}$ Varying the size of the hollow spheres would be possible within limits by varying the water/surfactant ratio or the amount of APTS. ${ }^{16}$ If a cationic surfactant system is used, one may make hollow spheres with mesoporous shells as the surfactant may be incorporated in the silica shell. ${ }^{17,18}$
Comparing processes $\mathrm{A}$ and $\mathrm{B}$, we find the difference lies in the size distribution of the products. For process A, the late addition of APTS apparently leads to a rigid shell which is a copy of the original interface of the $\mathrm{W} / \mathrm{O}$ droplet. In process $\mathrm{B}$, the early addition seems lead to a 'soft' shell where a kind of Oswald ripening is then allowed, leading to a heterogeneous spheres. After washing, small spheres become hollow and large spheres become yolk/shell. Thus in adjusting the order and the amount of APTS addition, we have developed a way to control the particle size and its interior filling extent.

In summary, we have designed a new method for making hollow nanospheres of silica containing a luminescent agent and superparamagnetic $\mathrm{Fe}_{3} \mathrm{O}_{4}$ nanoparticles. Their multifunctional utility as optical and MR imaging contrast agents for cell-labeling would be very useful in biomedical applications ${ }^{19-22}$ which we are further exploring.

This research was supported by a grant from the National Science Council of Taiwan. We thank Ms Yi-Chun Chuang for help with confocal microscopy measurements.

\section{Notes and references}

1 P. M. Arnal, M. Comotti and F. Schuth, Angew. Chem., Int. Ed., $2006,45,8224$.

2 X. F. Song and L. Gao, J. Phys. Chem. C, 2007, 111, 8180.

3 M. Chen, L. M. Wu, S. X. Zhou and B. You, Adv. Mater., 2006, 18, 801.

4 W. J. Li, X. X. Sha, W. J. Dong and Z. C. Wang, Chem. Commun., 2002, 2434.

5 H. J. Hah, J. S. Kim, B. J. Jeon, S. M. Koo and Y. E. Lee, Chem. Commun., 2003, 1712.

6 Y. Q. Yeh, B. C. Chen, H. P. Lin and C. Y. Tang, Langmuir, 2006, 22, 6 .

7 D. H. M. Buchold and C. Feldmann, Nano Lett., 2007, 7, 3489.

8 D. K. Yi, S. S. Lee, G. C. Papaefthymiou and J. Y. Ying, Chem. Mater., 2006, 18, 614.

9 J. Eastoe, B. H. Robinson, D. C. Steytler and D. Thornleeson, Adv. Colloid Interface Sci., 1991, 36, 1.

10 S. Santra, R. P. Bagwe, D. Dutta, J. T. Stanley, G. A. Walter, W. Tan, B. M. Moudgil and R. A. Mericle, Adv. Mater., 2005, 17, 2165 .

11 C. W. Lu, Y. Hung, J. K. Hsiao, M. Yao, T. H. Chung, Y. S. Lin, S. H. Wu, S. C. Hsu, H. M. Liu, C. Y. Mou, C. S. Yang, D. M. Huang and Y. C. Chen, Nano Lett., 2007, 7, 149.

12 L. Shao, D. Caruntu, J. F. Chen, C. J. O'Connor and W. L. Zhou, J. Appl. Phys., 2005, 97, 10Q908.

13 B. Peng, M. Chen, S. X. Zhou, L. M. Wu and X. H. Ma, J. Colloid Interface Sci., 2008, 321, 67.

14 R. Koole, M. M. van Schooneveld, J. Hilhorst, C. D. Donega D. C. t Hart, A. van Blaaderen, D. Vanmaekelbergh and A. Meijerink, Chem. Mater., 2008, 20, 2503.

15 S. Ikeda, Y. Ikoma, H. Kobayashi, T. Harada, T. Torimoto, B. Ohtani and M. Matsumura, Chem. Commun., 2007, 3753.

16 F. J. Arriagada and K. Osseo-Asare, J. Colloid Interface Sci., 1999, 211, 210.

17 Z. G. Feng, Y. S. Li, D. C. Niu, L. Li, W. R. Zhao, H. R. Chen, J. H. Gao, M. L. Ruan and J. L. Shi, Chem. Commun., 2008, 2629.

18 L. Zhang, S. Z. Qiao, Y. G. Jin, Z. G. Chen, H. C. Gu and G. Q. Lu, Adv. Mater., 2008, 20, 805.

19 W. J. Rieter, J. S. Kim, K. M. L. Taylor, H. Y. An, W. L. Lin, T. Tarrant and W. B. Lin, Angew. Chem., Int. Ed., 2007, 46, 3680.

20 Y. S. Lin, S. H. Wu, Y. Hung, Y. H. Chou, C. Chang, M. L. Lin, C. P. Tsai and C. Y. Mou, Chem. Mater., 2006, 18, 5170 .

21 C. P. Tsai, Y. Hung, Y. H. Chou, D. M. Huang, J. K. Hsiao, C. Chang, Y. C. Chen and C. Y. Mou, Small, 2008, 4, 186.

22 S. H. Wu, Y. S. Lin, Y. Hung, Y. H. Chou, Y. H. Hsu, C. Chang and C. Y. Mou, ChemBioChem, 2008, 9, 53. 